\title{
FITOSSOCIOLOGIA DE UMA FLORESTA PLUVIAL SUBTROPICAL PRIMÁRIA NO SUL DO BRASIL
}

\author{
João Paulo de Maçaneiro ${ }^{\text {1* }}$, Rafaela Cristina Seubert² ${ }^{2}$ Lauri Amândio Schorn ${ }^{2}$ \\ ${ }^{1 *}$ Universidade Federal do Paraná, Programa de Pós-Graduação em Engenharia Florestal, Curitiba, Paraná, Brasil - \\ jpmacaneiro@gmail.com \\ ${ }^{2}$ Universidade Regional de Blumenau, Departamento de Engenharia Florestal, Blumenau, Santa Catarina, Brasil - \\ rc.seubert@gmail.com; lauri.schorn@gmail.com \\ Recebido para publicação: 12/10/2014 - Aceito para publicação: 13/03/2014
}

\begin{abstract}
Resumo
Em Santa Catarina, a Floresta Pluvial Subtropical encontra-se alterada, sobretudo em sua composição e estrutura. Assim, trabalhos que caracterizem os remanescentes florestais são importantes, pois podem servir de base para estudos de meta-análise. Neste trabalho, foi caracterizada a composição e a estrutura de uma Floresta Pluvial Subtropical e comparou-se a composição de espécies dessa floresta com outras da mesma fitofisionomia. A vegetação foi amostrada em 25 parcelas de $400 \mathrm{~m}^{2}$, em que foram medidos os indivíduos com DAP $\geq 5 \mathrm{~cm}$. Foram amostrados 1.727 indivíduos, distribuídos em 144 espécies, 91 gêneros e 45 famílias. Myrtaceae, Lauraceae e Fabaceae foram as famílias mais ricas, com destaque para os gêneros Ocotea, Myrcia e Eugenia. As espécies com maior percentual de importância foram Euterpe edulis Mart., Sloanea guianensis (Aubl.) Benth., Ocotea aciphylla (Nees \& Mart.) Mez e Tapirira guianensis Aubl. Foram detectados dois grupos florísticos que se formaram devido à proximidade geográfica, sendo o primeiro grupo formado pelos estudos realizados na região sul-catarinense e o segundo por estudos realizados na região do Vale do Itajaí. Os resultados indicaram que a floresta é composta por espécies clímax tolerantes à sombra e pode ser caracterizada como um remanescente de vegetação bem conservado, indicando sua importância para conservação. Palavras-chave: Composição; estrutura; grupos ecológicos; similaridade; Vale do Itajaí.
\end{abstract}

\begin{abstract}
Phytosociology of a primary Subtropical Rain Forest in southern Brazil. The Subtropical Rain Forest is altered in Santa Catarina regarding its composition and structure. Thus, the studies that focus on these forest remnants are important because they can consist as a basis for meta-analysis. In this work research we intend to characterize the composition and structure of a Subtropical Rain Forest stand and compare the species composition of this stand with others in the same forest type. The vegetation was sampled through 25 sample plots of $400 \mathrm{~m}^{2}$, where individuals with DBH $\geq 5 \mathrm{~cm}$ were measured. We sampled 1,727 individuals belonging to 144 species, 91 genus and 45 families. Myrtaceae, Lauraceae and Fabaceae were the richest families, especially the Ocotea, Eugenia and Myrcia genus. The species with the highest importance percentual were Euterpe edulis Mart., Sloanea guianensis (Aubl.) Benth., Ocotea aciphylla (Nees \& Mart.) Mez, Tapirira guianensis Aubl. We detected two floristic groups formed due to geographical proximity; the first was formed by studies conducted in the southern region and the second by studies conducted in the Itajaí Valley. Our results indicated that the studied forest stand is composed primarily of shade-tolerant climax species and can be characterized as a well-preserved forest remnant. The results indicate the importance of this area for conservation.
\end{abstract}

Keywords: Composition; structure; ecological groups; similarity; Itajaí Valley.

\section{INTRODUÇÃO}

Devido a sua variedade de ecossistemas, o Brasil é um dos países com maior diversidade de espécies vegetais do mundo, apresentando mais de 45 mil espécies de plantas conhecidas (FORZZA et al., 2014), o que corresponde a cerca de 13\% da flora mundial, estimada atualmente em 352.000 espécies de plantas (THE PLANT LIST, 2014). Desse total, o domínio da Mata Atlântica abriga cerca de 19.335 
espécies de plantas vasculares, das quais 39,5\% são endêmicas (FORZZA et al., 2012). Nos últimos anos, alguns estudos revelaram uma diversidade de espécies particularmente elevada para o domínio da Mata Atlântica, como é o caso das 476 espécies de plantas registradas em um hectare na Estação Biológica de Santa Lúcia, no Espírito Santo, e 454 espécies na Serra do Canduru, no sul da Bahia (GIULIETTI et al., 2005). Entretanto, a Mata Atlântica tem sido alvo de intenso processo de fragmentação, o que ocasionou a redução de muitas áreas ocupadas por vegetação nativa e alarmantes taxas de perdas de biodiversidade e dos recursos naturais e genéticos (MYERS et al., 2000).

A Mata Atlântica sensu stricto (OLIVEIRA-FILHO; FONTES, 2000) representa uma região heterogênea com diferentes fitofisionomias vegetacionais e elevada riqueza de espécies que, por vezes, superam a das florestas equatoriais amazônicas (THOMAS et al., 1998). Entre as fitofisionomias da Mata Atlântica com maior diversidade de espécies está a Floresta Pluvial Subtropical (OLIVEIRA-FILHO, 2009). Essas florestas são caracterizadas pela presença de estratos bem definidos, com fanerófitos subdivididos nas formas de vida macro, meso, micro e nanofanerófitos, além das lianas lenhosas e epífitas em abundância que a diferenciam das outras fitofisionomias vegetacionais (KLEIN, 1980). No estado de Santa Catarina, a Floresta Pluvial Subtropical cobria originalmente $29.282 \mathrm{~km}^{2}$, o que equivale a $31 \%$ do território catarinense. Atualmente, estudos realizados pelo Inventário Florístico Florestal de Santa Catarina registraram uma cobertura remanescente de 40,4\% da Floresta Pluvial Subtropical no estado, sendo que 74\% desse total se encontram em fragmentos com até 50 hectares e 7,2\% da área pertencem a fragmentos com área superior a 1.000 ha (VIBRANS et al., 2013).

Nos últimos anos, em função da atual situação de degradação da Mata Atlântica em Santa Catarina, vários trabalhos foram desenvolvidos com o propósito de caracterizar a composição e estrutura de remanescentes florestais. A maior parte desses trabalhos foi realizada em escalas locais (CITADITIZANETTE, 1995; VIBRANS, 1999; IZA, 2002; SEVEGNANI, 2003; GHODDOSI, 2005; MARTINS, 2005; NEGRELLE, 2006; SILVA, 2006; COLONETTI et al., 2009; EMERICH, 2009; SCHORN; GALVÃO, 2009; MARTINS, 2010), sendo poucos trabalhos em escalas mais amplas (LINGNER et al., 2013). Apesar de a maioria dos trabalhos terem sido realizados em escalas locais, eles servem de base para determinar padrões de distribuição das espécies ou associações florísticas em estudos de metaanálise, além de possibilitarem a geração de novas hipóteses sobre a relação entre as espécies arbóreoarbustivas e as condições ambientais, as quais não seriam possíveis sem a soma de vários trabalhos já publicados (MORO; MARTINS, 2011).

Devido à expansão urbana nos municípios de Santa Catarina, a fragmentação florestal ocorre, na maioria das vezes, devido à substituição de parte da floresta para a implantação de loteamentos urbanos ou áreas industriais. Esse processo refletiu na diminuição da cobertura florestal da área urbana do município de Brusque, sendo que grande parte dos remanescentes florestais bem conservados ficaram restritos às áreas de encosta com relevo íngreme e de difícil acesso. A Reserva Particular do Patrimônio Natural (RPPN) Chácara Edith, localizada em Brusque, SC, local do presente estudo, é uma das poucas áreas onde é possível encontrar remanescentes de Floresta Pluvial Subtropical bem conservados dentro de área urbana no estado de Santa Catarina. Essa reserva é uma das áreas prioritárias para conservação em Santa Catarina e está inserida na zona núcleo da Reserva da Biosfera da Mata Atlântica (RBMA). Além disso, a RPPN Chácara Edith é considerada o único Posto Avançado da RBMA no estado, o que demonstra seu alto valor para conservação da diversidade biológica. No entanto, são escassos os estudos voltados à flora arbóreo-arbustiva nessa reserva. Assim, o presente estudo teve como objetivos: (i) caracterizar a composição e estrutura do estrato arbóreo-arbustivo de uma Floresta Pluvial Subtropical em Santa Catarina; e (ii) comparar a composição de espécies do presente estudo com outros realizados na Floresta Pluvial Subtropical de Santa Catarina.

\section{MATERIAL E MÉTODOS}

A área de estudo está inserida na bacia hidrográfica do rio Itajaí, em Brusque, SC. Pertence à RPPN Chácara Edith, que possui área total de 415,8 ha e altitude que varia de 20 a $300 \mathrm{~m}$, estando localizada entre $27^{\circ} 05^{\prime}$ ' e $27^{\circ} 06^{\prime} \mathrm{S}$ e $48^{\circ} 51^{\prime}$ ' e $48^{\circ} 54^{\prime}$ 'W.

A vegetação na área de estudo é constituída pela Floresta Pluvial Subtropical Submontana de Encosta (OLIVEIRA-FILHO, 2009), daqui em diante referida como Floresta Pluvial Subtropical. Em 1923, o proprietário da reserva iniciou a exploração madeireira na área de estudo, que durou até 1930. 
Essa exploração teve como foco a canela-preta (Ocotea catharinensis Mez) e outras espécies de interesse econômico. Atualmente, o estado de conservação da floresta na área de estudo pode ser classificado como vegetação primária, porém alterada devido à retirada de madeiras de lei até 1930.

O clima do município de Brusque, segundo a classificação de Köppen, é do tipo Cfa - clima subtropical úmido sem estação seca e com verão quente. Sua temperatura média anual varia entre 19 e $20{ }^{\circ} \mathrm{C}$, com médias mensais variando entre $15{ }^{\circ} \mathrm{C}$ no mês mais frio (julho) e $25^{\circ} \mathrm{C}$ nos meses mais quentes (janeiro e fevereiro). A umidade relativa anual varia entre $84-86 \%$, com médias mensais entre $80 \%$ no mês mais quente (dezembro) e $88 \%$ nos meses mais frios (junho e julho) (EMPRESA DE PESQUISA AGROPECUÁRIA E EXTENSÃO RURAL DE SANTA CATARINA (EPAGRI), 2002). Dados coletados pela Estação Brusque da Agência Nacional de Águas $\left(27^{\circ} 06^{\prime} 02,16\right.$ " S, 48 $55^{\prime} 04,08^{\prime \prime}$ W e $25 \mathrm{~m}$ de altitude), distante $2,8 \mathrm{~km}$ da área de estudo, registraram, no período de 1941 a 2013, precipitações totais anuais que variaram de 460 a $3.004 \mathrm{~mm}$, com média de $1.568 \mathrm{~mm}$.

A geologia da região é formada pelo Complexo Metamórfico Brusque, composto pela Formação Botuverá, pelo Granodiorito Valsungana e pelo Granito Guabiruba (SCHULZ-JÚNIOR; ALBUQUERQUE, 1969). Essa formação é constituída por uma associação metavulcano-sedimentar composta por filitos e xistos micáceos intercalados com semipelitos, quartzitos, rochas cálci-silicáticas e mármores, entre outros (PHILIPP et al., 2004). Predomina, na região de estudo, uma associação entre dois tipos de solos: em relevos ondulados, o CAMBISSOLO HÁPLICO Tb A moderado de textura argilosa, e, em relevo forte ondulado e montanhoso, o NEOSSOLO LITÓLICO Distrófico A moderado de textura argilosa (EMPRESA BRASILEIRA DE PESQUISA AGROPECUÁRIA (EMBRAPA), 2004).

Para a coleta dos dados, foram utilizadas parcelas dispostas em faixas, com o objetivo de representar a maior variação da vegetação possível ao longo da encosta analisada. As parcelas foram dispostas em cinco faixas, todas iniciando na base da encosta e terminando no seu topo. No total, foram distribuídas sistematicamente 25 parcelas de $20 \times 20 \mathrm{~m}\left(400 \mathrm{~m}^{2}\right)$, totalizando $10.000 \mathrm{~m}^{2}$ de área amostral. Essas parcelas foram distanciadas por aproximadamente $50 \mathrm{~m}$ entre si e $25 \mathrm{~m}$ entre as faixas. Cada parcela foi subdividida em quatro subparcelas de $10 \times 10 \mathrm{~m}\left(100 \mathrm{~m}^{2}\right)$. Em cada parcela, foram registrados os indivíduos arbóreo-arbustivos vivos com DAP $\geq 5 \mathrm{~cm}$ e sua posição sociológica (considerou-se como inseridos no estrato superior da floresta os indivíduos cuja altura de copa não foi superada por nenhuma outra árvore; inseridos no estrato médio estavam os indivíduos cuja copa foi superada por pelo menos uma árvore; inseridos no estrato inferior estavam os indivíduos cuja copa foi superada por duas ou mais árvores).

O material botânico coletado foi identificado por comparação com fotografias digitais nos sítios da Flora Digital (http://ufrgs.br/floradigital) e do INCT (http://inct.splink.org.br), com exsicatas depositadas no Herbário Dr. Roberto Miguel Klein, da Universidade Regional de Blumenau (FURB), e, também, mediante consulta à literatura taxonômica e aos especialistas da FURB.

As espécies foram agrupadas em famílias (APG III, 2009) e por grupo ecológico, adotando-se a metodologia sugerida por Oliveira-Filho (1994) nas seguintes categorias: pioneira (P), clímax exigente de luz (CL) e clímax tolerante à sombra (CS).

Para descrever a estrutura da vegetação, foram calculados os parâmetros fitossociológicos clássicos de Mueller-Dombois e Ellenberg (2002), quais sejam, densidade, dominância e frequência absolutas e relativas e o percentual de importância para cada espécie. Nessa análise, foram consideradas as subparcelas de $10 \times 10 \mathrm{~m}$.

Para verificar a similaridade florística do presente estudo com outros realizados na Floresta Pluvial Subtropical de Santa Catarina, aplicaram-se dois métodos multivariados a partir de uma matriz de presença e ausência de espécies. O primeiro deles foi uma análise de agrupamentos através do método UPGMA, utilizando-se o coeficiente de similaridade de Sørensen, por ser recomendável para dados qualitativos (LEGENDRE; LEGENDRE, 2012). Em seguida, calculou-se o coeficiente de correlação cofenética, que mede o grau de distorção entre a matriz original e a matriz calculada, para obtenção do dendrograma (LEGENDRE; LEGENDRE, 2012). A significância estatística entre a matriz de distâncias e a matriz cofenética foi verificada por meio do teste de Mantel (MANTEL, 1967). Em seguida, foi realizado um Escalonamento Multidimensional Não Métrico (NMDS), com o objetivo de investigar como os diferentes estudos se ordenavam no espaço multidimensional da composição de espécies. Foi utilizada a distância de Sørensen para a matriz de presença e ausência de espécies e calculou-se uma medida de stress, que indica a proporção da variância das disparidades não explicadas pelo modelo NMDS (McCUNE; GRACE, 2002). A

FLORESTA, Curitiba, PR, v. 45, n. 3, p. 555 - 566, jul. / set. 2015.

Maçaneiro, J. P. de; Seubert, R. C.; Schorn, L. A.

ISSN eletrônico 1982-4688 / ISSN impresso 0015-3826

DOI: $10.5380 /$ rf.v45i3.38157 
significância estatística do stress foi testada por meio do teste de Monte Carlo e a confirmação da estabilidade do stress na porção final das iterações foi verificada conforme McCune e Grace (2002). Por fim, foi testada a significância estatística dos grupos florísticos formados pela análise de agrupamentos e pelo NMDS, por meio da ANOSIM (CLARKE, 1993).

\section{RESULTADOS E DISCUSSÃO}

Foram amostrados 1.727 indivíduos, distribuídos em 144 espécies, 91 gêneros e 45 famílias (Tabela 1). Das espécies registradas, quatro constam na Lista Oficial das Espécies da Flora Brasileira Ameaçadas de Extinção (MINISTÉRIO DO MEIO AMBIENTE (MMA), 2014): Ocotea catharinensis Mez (canela-preta), Ocotea odorifera (Vell.) Rohwer (canela-sassafrás), Euterpe edulis Mart. (palmiteiro) e Cedrela fissilis Vell. (cedro).

As famílias botânicas que se destacaram em número de espécies foram Myrtaceae (34 espécies), Lauraceae (19), Fabaceae (10), Rubiaceae (oito) e Melastomataceae (sete), as quais corresponderam a $54,2 \%$ do total de espécies. Essas famílias são consideradas as mais ricas em número de espécies da Floresta Pluvial Subtropical (OLIVEIRA-FILHO; FONTES, 2000; SCHEER; BLUM, 2011; LINGNER et al., 2013). O fato de Myrtaceae e Lauraceae se destacarem entre as famílias com maior riqueza de espécies na área de estudo pode sugerir que a floresta encontra-se em ótimo estado de conservação da sua composição florística.

Os gêneros com maior riqueza de espécies foram Ocotea (12 espécies), Myrcia (10) e Eugenia (nove), contribuindo com $22 \%$ do total de espécies registradas. Esses gêneros já foram apontados como os mais representativos em uma compilação de diversos estudos realizados em diferentes regiões do domínio da Mata Atlântica por Oliveira-Filho e Fontes (2000). De forma semelhante, estudos realizados por Scheer e Blum (2011) no estado do Paraná e por Lingner et al. (2013) em Santa Catarina também destacaram os gêneros Eugenia, Myrcia e Ocotea como os mais ricos em espécies da Floresta Pluvial Subtropical.

O grupo ecológico mais representativo foi o das clímax tolerantes à sombra, que corresponderam a 100 espécies $(69,4 \%)$, seguindo-se das clímax exigentes de luz, com 40 espécies $(27,8 \%)$, e das pioneiras, com apenas quatro espécies $(2,8 \%)$. Entre as espécies pioneiras amostradas estão Clethra scabra Pers. e Clusia criuva Cambess., que foram registradas nas parcelas inseridas no topo da encosta. Essa constatação pode ser explicada pelo fato de o topo da encosta ser constituído por solo mais raso, às vezes rochoso e de rápida drenagem, o que condiciona a formação de uma vegetação mais aberta, homogênea e menos desenvolvida (KLEIN, 1980). Assim, o ambiente proporcionado pelo topo da encosta pode refletir em condições ambientais favoráveis para o estabelecimento dessas espécies na floresta. Contudo, as outras espécies pioneiras amostradas - Cecropia glaziovii Snethl. e Tibouchina mutabilis (Vell.) Cogn. - foram encontradas em parcelas inseridas na base da encosta, possivelmente em áreas cicatrizadas por antigas clareiras causadas pela queda de árvores no interior da floresta. Essas espécies também foram apontadas por Tabarelli e Mantovani (1999) como sendo típicas de clareiras em processo de regeneração, em estudos sobre a colonização de clareiras naturais na Floresta Pluvial Subtropical da Serra do Mar de São Paulo. De forma geral, o fato de as espécies clímax tolerantes à sombra serem predominantes na área de estudo sugere que a floresta encontra-se em ótimas condições naturais de conservação.

A densidade de indivíduos da floresta foi de 1.727 ind.ha $^{-1}$ e as espécies mais representativas foram Euterpe edulis Mart., Sloanea guianensis (Aubl.) Benth., Garcinia gardneriana (Planch. \& Triana) Zappi, Cyathea corcovadensis (Raddi) Domin e Ocotea aciphylla (Nees \& Mart.) Mez, as quais somaram $43,2 \%$ da densidade total (Tabela 2). Além disso, a densidade de indivíduos registrada no presente estudo está dentro do encontrado em outros trabalhos realizados na Floresta Pluvial Subtropical de Santa Catarina, cuja faixa de variação está entre 1.412 e 2.191 ind.ha $^{-1}$ (CITADINI-ZANETTE, 1995; VIBRANS, 1999; IZA, 2002; SEVEGNANI, 2003; GHODDOSI, 2005; NEGRELLE, 2006; SILVA, 2006; COLONETTI et al., 2009; SCHORN; GALVÃO, 2009).

A frequência absoluta foi relativamente baixa para a maioria das espécies. Apenas E. edulis e $S$. guianensis apresentaram frequência superior a 50\%, ou seja, ocorreram em mais da metade das subparcelas amostradas. Esse resultado indica que essas espécies apresentam dispersão bastante ampla na floresta estudada, resultado semelhante ao encontrado por outros autores em florestas bem conservadas da mesma região (VELOSO; KLEIN, 1959; KLEIN, 1980; SEVEGNANI, 2003; GHODDOSI, 2005). Com frequência entre 20 e 50\%, foram registradas somente 10 espécies (6,9\%), enquanto que 132 espécies $(91,7 \%)$ 
apresentaram frequência inferior a 20\%, demonstrando que grande parte das espécies se restringiu a ocorrer em poucas subparcelas, pois 38 espécies $(26,4 \%)$ ocorreram em apenas uma subparcela do levantamento.

A área basal total da floresta foi de $40,39 \mathrm{~m}^{2} \cdot \mathrm{ha}^{-1}$, sendo que as espécies Sloanea guianensis, Euterpe edulis, Tapirira guianensis Aubl., Ocotea aciphylla e Vantanea compacta (Schnizl.) Cuatrec. contribuíram com 32,9\% desse total. Esse valor pode ser considerado alto quando comparado a outros remanescentes de Floresta Pluvial Subtropical Submontana de Santa Catarina, cuja faixa de variação está entre 27,94 e 39,85 m². $\mathrm{ha}^{-1}$ (CITADINI-ZANETTE, 1995; VIBRANS, 1999; SEVEGNANI, 2003; GHODDOSI, 2005; SILVA, 2006; COLONETTI et al., 2009; MARTINS, 2005; 2010), indicando que, mesmo após a exploração madeireira ocorrida até 1930, a floresta encontra-se em fase muito adiantada na reconstrução de sua estrutura.

Verificou-se que $E$. edulis foi a espécie com maior percentual de importância na área de estudo ( $\mathrm{PI}=13,5 \%$ ), principalmente pelos seus elevados valores de densidade e frequência (Tabela 1). Em relação à posição sociológica, grande parte de seus indivíduos se concentrou no estrato inferior da floresta, onde foram registrados 289 ind.ha $^{-1}$, seguindo-se do estrato médio (143) e do estrato superior (10). Em áreas bem conservadas da Floresta Pluvial Subtropical de Santa Catarina, vários estudos têm apontado E. edulis como a espécie de maior densidade no interior das florestas (VELOSO; KLEIN, 1959; VIBRANS, 1999; IZA, 2002; SEVEGNANI, 2003; COLONETTI et al., 2009; SCHORN; GALVÃO, 2009). Klein (1980), por exemplo, menciona que as maiores densidades dessa espécie ocorriam em áreas com solos muito úmidos, onde podia ultrapassar 1.000 ind.ha $^{-1}$. No entanto, o mesmo autor também verificou que E. edulis ocorria com elevada densidade nas florestas bem conservadas das encostas da região do Vale do Itajaí, SC, se tornando muitas vezes a única espécie dominante do estrato médio dessas florestas.

Outra espécie que se destacou por apresentar o segundo maior percentual de importância foi $S$. guianensis (PI $=6,8 \%$ ). Essa espécie já foi apontada em estudos históricos como subdominante no estrato superior das florestas bem conservadas do Vale do Itajaí (KLEIN, 1980) e junto com $O$. catharinensis caracterizava o dossel das comunidades primárias situadas em grande parte das encostas das Serras do Mar e do Vale do Itajaí em Santa Catarina (VELOSO; KLEIN, 1959). Neste estudo, S. guianensis se destacou por apresentar valores fitossociológicos elevados na comunidade, além de somar a maior dominância da floresta ( $\mathrm{DoR}=8,6 \%)$. Constatou-se, ainda, que seus indivíduos se concentraram no estrato médio da floresta, onde foram registrados 61 ind.ha $^{-1}$, seguindo-se do estrato inferior (35) e do estrato superior (11). De acordo com Veloso e Klein (1959), essa espécie apresentava característica de dominante indiferente, exercendo essas funções em virtude de sua grande adaptação aos diferentes hábitats encontrados na floresta, tornando-se, assim, uma das espécies mais importantes no estrato médio das florestas bem conservadas na região estudada.

A espécie $O$. aciphylla se caracterizou por apresentar a maior parte de seus indivíduos no estrato médio da floresta (32 ind.ha ${ }^{-1}$ ), seguindo-se do estrato superior (18) e do estrato inferior (15). Klein (1980) menciona que essa espécie era muito abundante nas florestas primárias situadas nos topos das encostas na região do Vale do Itajaí, SC, possuindo muitos indivíduos jovens no interior dessas florestas. Além disso, Veloso e Klein (1959), em seus estudos na Mata do Hoffmann, local do presente estudo, mencionam que $O$. aciphylla se limitava às áreas mais secas da encosta, apresentando árvores de copas menores, mas com muitos indivíduos jovens, motivo pelo qual, no estrato superior da floresta, sua dominância era menor.

As espécies $V$. compacta e T. guianensis se destacaram por apresentarem indivíduos de porte agigantado e com copas largas e densifoliadas, caracterizando o estrato superior, respectivamente, com $14 \mathrm{e}$ 21 ind.ha $^{-1}$, seguindo-se do estrato médio (8 e 14) e inferior (4 e 1). Veloso e Klein (1959) mencionam $V$. compacta como uma espécie seletiva xerófita que ocorria com elevada densidade nas florestas primárias situadas nos topos das encostas íngremes. No entanto, pelo fato de essa espécie apresentar apenas indivíduos adultos e velhos, os autores concluíram que ela não estaria mais encontrando um ambiente favorável para o seu desenvolvimento e poderia estar sendo substituída por outras espécies mais exigentes em condições ambientais. Já a espécie T. guianensis, Klein (1980) a caracterizou como dominante em todas as comunidades estabelecidas no alto das encostas do Vale do Itajaí. Nesses ambientes, T. guianensis encontrou o seu ótimo de ocorrência, apresentando tanto indivíduos adultos no estrato superior quanto indivíduos jovens nos estratos médio e inferior das florestas. No presente estudo, registrou-se, para T. guianensis, apenas 1 ind.ha $^{-1}$ no estrato inferior da floresta, sugerindo que essa espécie pode estar

FLORESTA, Curitiba, PR, v. 45, n. 3, p. 555 - 566, jul. / set. 2015. 
sofrendo com taxas de recrutamento ou mortalidade de indivíduos. No entanto, estudos sobre a dinâmica populacional dessa espécie devem ser realizados para confirmar essa hipótese.

Tabela 1. Parâmetros fitossociológicos calculados para as espécies do estrato arbóreo-arbustivo de uma Floresta Pluvial Subtropical no Sul do Brasil.

Table 1. Phytosociological parameters calculated for the species of the tree-shrub layer of an Subtropical Rain Forest in southern Brazil.

\begin{tabular}{|c|c|c|c|c|c|c|c|c|c|}
\hline Família & Espécie & GE & DA & DR & DoA & DoR & FA & FR & PI \\
\hline Anacardiaceae & Tapirira guianensis Aubl. & CL & 36 & 2,08 & 2,52 & 6,25 & 23,00 & 2,18 & 3,51 \\
\hline \multirow[t]{3}{*}{ Annonaceae } & Duguetia lanceolata A.St.-Hil. & CS & 12 & 0,69 & 0,55 & 1,36 & 10,00 & 0,95 & 1,00 \\
\hline & Guatteria australis A.St.-Hil. & $\mathrm{CS}$ & 20 & 1,16 & 0,25 & 0,63 & 17,00 & 1,61 & 1,13 \\
\hline & Xylopia brasiliensis Spreng. & CL & 18 & 1,04 & 0,82 & 2,03 & 16,00 & 1,52 & 1,53 \\
\hline \multirow[t]{3}{*}{ Apocynaceae } & Aspidosperma australe Müll.Arg. & CL & 9 & 0,52 & 0,99 & 2,44 & 9,00 & 0,85 & 1,27 \\
\hline & Aspidosperma tomentosum Mart. & $\mathrm{CL}$ & 3 & 0,17 & 0,32 & 0,79 & 3,00 & 0,28 & 0,42 \\
\hline & Tabernaemontana catharinensis A.DC. & $\mathrm{CL}$ & 1 & 0,06 & 0,02 & 0,05 & 1,00 & 0,09 & 0,07 \\
\hline \multirow[t]{2}{*}{ Aquifoliaceae } & Ilex paraguariensis A.St.-Hil. & $\mathrm{CS}$ & 1 & 0,06 & 0,002 & 0,01 & 1,00 & 0,09 & 0,05 \\
\hline & Ilex theezans Mart. ex Reissek & $\mathrm{CL}$ & 8 & 0,46 & 0,09 & 0,23 & 8,00 & 0,76 & 0,48 \\
\hline \multirow[t]{2}{*}{ Araliaceae } & Schefflera angustissima (Marchal) Frodin & CL & 2 & 0,12 & 0,05 & 0,11 & 2,00 & 0,19 & 0,14 \\
\hline & Schefflera morototoni (Aubl.) Maguire et al. & CL & 6 & 0,35 & 0,24 & 0,58 & 6,00 & 0,57 & 0,50 \\
\hline Arecaceae & Euterpe edulis Mart. & $\mathrm{CS}$ & 442 & 25,59 & 2,78 & 6,87 & 84,00 & 7,97 & 13,48 \\
\hline \multirow[t]{2}{*}{ Bignoniaceae } & Cybistax antisyphilitica (Mart.) Mart. & CS & 1 & 0,06 & 0,01 & 0,02 & 1,00 & 0,09 & 0,06 \\
\hline & Jacaranda micrantha Cham. & CL & 1 & 0,06 & 0,02 & 0,05 & 1,00 & 0,09 & 0,07 \\
\hline Burseraceae & Protium kleinii Cuatrec. & CS & 5 & 0,29 & 0,65 & 1,62 & 4,00 & 0,38 & 0,76 \\
\hline Celastraceae & Maytenus gonoclada Mart. & $\mathrm{CS}$ & 13 & 0,75 & 0,11 & 0,27 & 13,00 & 1,23 & 0,75 \\
\hline Chrysobalanaceae & Hirtella hebeclada Moric. ex DC. & CS & 11 & 0,64 & 0,39 & 0,97 & 10,00 & 0,95 & 0,85 \\
\hline Clethraceae & Clethra scabra Pers. & $\mathrm{P}$ & 2 & 0,12 & 0,02 & 0,04 & 1,00 & 0,09 & 0,08 \\
\hline \multirow[t]{2}{*}{ Clusiaceae } & Clusia criuva Cambess. & $\mathrm{P}$ & 2 & 0,12 & 0,05 & 0,12 & 2,00 & 0,19 & 0,14 \\
\hline & Garcinia gardneriana (Planch. \& Triana) Zappi & $\mathrm{CS}$ & 66 & 3,82 & 0,35 & 0,87 & 40,00 & 3,80 & 2,83 \\
\hline Cunoniaceae & Weinmannia paulliniifolia Pohl ex Ser. & CL & 1 & 0,06 & 0,01 & 0,02 & 1,00 & 0,09 & 0,06 \\
\hline \multirow[t]{3}{*}{ Cyatheaceae } & Alsophila setosa Kaulf. & CS & 37 & 2,14 & 0,17 & 0,42 & 18,00 & 1,71 & 1,42 \\
\hline & Cyathea corcovadensis (Raddi) Domin & $\mathrm{CS}$ & 66 & 3,82 & 0,35 & 0,87 & 33,00 & 3,13 & 2,61 \\
\hline & Cyathea delgadii Sternb. & $\mathrm{CS}$ & 53 & 3,07 & 0,25 & 0,62 & 32,00 & 3,04 & 2,24 \\
\hline Elaeocarpaceae & Sloanea guianensis (Aubl.) Benth. & $\mathrm{CS}$ & 107 & 6,20 & 3,49 & 8,65 & 59,00 & 5,60 & 6,81 \\
\hline \multirow[t]{3}{*}{ Euphorbiaceae } & Actinostemon concolor (Spreng.) Müll.Arg. & $\mathrm{CS}$ & 17 & 0,98 & 0,08 & 0,21 & 13,00 & 1,23 & 0,81 \\
\hline & Alchornea triplinervia (Spreng.) Müll.Arg. & CL & 29 & 1,68 & 1,97 & 4,88 & 19,00 & 1,80 & 2,79 \\
\hline & Aparisthmium cordatum (A.Juss.) Baill. & CL & 54 & 3,13 & 0,33 & 0,82 & 33,00 & 3,13 & 2,36 \\
\hline \multirow[t]{10}{*}{ Fabaceae } & $\begin{array}{l}\text { Abarema langsdorffii (Benth.) Barneby \& } \\
\text { J.W.Grimes }\end{array}$ & CL & 4 & 0,23 & 0,14 & 0,34 & 4,00 & 0,38 & 0,32 \\
\hline & Andira fraxinifolia Benth. & $\mathrm{CL}$ & 8 & 0,46 & 0,06 & 0,15 & 7,00 & 0,66 & 0,43 \\
\hline & Copaifera trapezifolia Hayne & $\mathrm{CS}$ & 13 & 0,75 & 0,72 & 1,79 & 13,00 & 1,23 & 1,26 \\
\hline & Dalbergia brasiliensis Vogel & $\mathrm{CL}$ & 1 & 0,06 & 0,04 & 0,10 & 1,00 & 0,09 & 0,08 \\
\hline & Inga sellowiana Benth. & CL & 4 & 0,23 & 0,20 & 0,50 & 4,00 & 0,38 & 0,37 \\
\hline & Machaerium hirtum (Vell.) Stellfeld & $\mathrm{CL}$ & 1 & 0,06 & 0,01 & 0,02 & 1,00 & 0,09 & 0,06 \\
\hline & Myrocarpus frondosus Allemão & $\mathrm{CL}$ & 1 & 0,06 & 0,02 & 0,04 & 1,00 & 0,09 & 0,06 \\
\hline & Ormosia arborea (Vell.) Harms & $\mathrm{CS}$ & 1 & 0,06 & 0,003 & 0,01 & 1,00 & 0,09 & 0,05 \\
\hline & Pterocarpus rohrii Vahl & CS & 7 & 0,41 & 0,42 & 1,04 & 7,00 & 0,66 & 0,70 \\
\hline & Zollernia ilicifolia (Brongn.) Vogel & CS & 1 & 0,06 & 0,04 & 0,11 & 1,00 & 0,09 & 0,09 \\
\hline Humiriaceae & Vantanea compacta (Schnizl.) Cuatrec. & CS & 26 & 1,51 & 2,14 & 5,29 & 18,00 & 1,71 & 2,83 \\
\hline \multirow[t]{17}{*}{ Lauraceae } & Aiouea saligna Meisn. & $\mathrm{CS}$ & 1 & 0,06 & 0,004 & 0,01 & 1,00 & 0,09 & 0,05 \\
\hline & Aniba firmula (Nees \& Mart.) Mez & CS & 6 & 0,35 & 0,08 & 0,21 & 6,00 & 0,57 & 0,37 \\
\hline & Cryptocarya mandioccana Meisn. & $\mathrm{CS}$ & 24 & 1,39 & 1,02 & 2,52 & 22,00 & 2,09 & 2,00 \\
\hline & Cryptocarya sp. & CS & 4 & 0,23 & 0,14 & 0,36 & 3,00 & 0,28 & 0,29 \\
\hline & Nectandra oppositifolia Nees & CL & 3 & 0,17 & 0,26 & 0,65 & 3,00 & 0,28 & 0,37 \\
\hline & Ocotea aciphylla (Nees \& Mart.) Mez & CS & 65 & 3,76 & 2,36 & 5,84 & 32,00 & 3,04 & 4,21 \\
\hline & Ocotea bicolor Vattimo-Gil & CS & 3 & 0,17 & 0,13 & 0,32 & 3,00 & 0,28 & 0,26 \\
\hline & Ocotea catharinensis $\mathrm{Mez}$ & CS & 20 & 1,16 & 1,44 & 3,58 & 18,00 & 1,71 & 2,15 \\
\hline & Ocotea dispersa (Nees \& Mart.) Mez & CL & 2 & 0,12 & 0,07 & 0,18 & 2,00 & 0,19 & 0,16 \\
\hline & Ocotea elegans $\mathrm{Mez}$ & CS & 14 & 0,81 & 0,41 & 1,03 & 11,00 & 1,04 & 0,96 \\
\hline & Ocotea lanata (Nees \& Mart.) Mez & CS & 1 & 0,06 & 0,003 & 0,01 & 1,00 & 0,09 & 0,05 \\
\hline & Ocotea mandioccana A.Quinet & $\mathrm{CS}$ & 1 & 0,06 & 0,32 & 0,79 & 1,00 & 0,09 & 0,32 \\
\hline & Ocotea nectandrifolia $\mathrm{Mez}$ & CS & 3 & 0,17 & 0,52 & 1,30 & 3,00 & 0,28 & 0,59 \\
\hline & Ocotea odorifera (Vell.) Rohwer & CS & 14 & 0,81 & 0,44 & 1,10 & 11,00 & 1,04 & 0,98 \\
\hline & Ocotea pulchra Vattimo-Gil & CS & 12 & 0,69 & 0,24 & 0,60 & 9,00 & 0,85 & 0,72 \\
\hline & Ocotea silvestris Vattimo-Gil & CS & 1 & 0,06 & 0,03 & 0,07 & 1,00 & 0,09 & 0,07 \\
\hline & Ocotea $\mathrm{sp}$ & $\mathrm{CS}$ & 2 & 0,12 & 0,01 & 0,02 & 2,00 & 0,19 & 0,11 \\
\hline
\end{tabular}




\begin{tabular}{|c|c|c|c|c|c|c|c|c|c|}
\hline & Persea major (Meisn.) L.E.Kopp & $\mathrm{CS}$ & 1 & 0,06 & 0,01 & 0,03 & 1,00 & 0,09 & 0,06 \\
\hline & Persea venosa Nees \& Mart. & $\mathrm{CS}$ & 1 & 0,06 & 0,11 & 0,28 & 1,00 & 0,09 & 0,15 \\
\hline Magnoliaceae & Magnolia ovata (A.St.-Hil.) Spreng. & CS & 3 & 0,17 & 0,10 & 0,25 & 3,00 & 0,28 & 0,24 \\
\hline Malpighiaceae & Byrsonima ligustrifolia A.Juss. & $\mathrm{CL}$ & 26 & 1,51 & 0,48 & 1,18 & 23,00 & 2,18 & 1,62 \\
\hline Malvaceae & Pseudobombax grandiflorum (Cav.) A.Robyns & CL & 4 & 0,23 & 1,19 & 2,94 & 4,00 & 0,38 & 1,18 \\
\hline \multirow[t]{7}{*}{ Melastomataceae } & Henriettella glabra (Vell.) Cogn. & CS & 5 & 0,29 & 0,06 & 0,16 & 4,00 & 0,38 & 0,27 \\
\hline & Miconia budlejoides Triana & CL & 3 & 0,17 & 0,02 & 0,05 & 3,00 & 0,28 & 0,17 \\
\hline & Miconia cabucu Hoehne & CL & 2 & 0,12 & 0,11 & 0,27 & 2,00 & 0,19 & 0,19 \\
\hline & Miconia cubatanensis Hoehne & $\mathrm{CL}$ & 1 & 0,06 & 0,002 & 0,01 & 1,00 & 0,09 & 0,05 \\
\hline & Miconia pusilliflora (DC.) Naudin & CL & 1 & 0,06 & 0,01 & 0,02 & 1,00 & 0,09 & 0,06 \\
\hline & Mouriri chamissoana Cogn. & CS & 1 & 0,06 & 0,002 & 0,01 & 1,00 & 0,09 & 0,05 \\
\hline & Tibouchina mutabilis (Vell.) Cogn. & $\mathrm{P}$ & 1 & 0,06 & 0,01 & 0,03 & 1,00 & 0,09 & 0,06 \\
\hline \multirow[t]{3}{*}{ Meliaceae } & Cabralea canjerana (Vell.) Mart. & CS & 20 & 1,16 & 1,24 & 3,06 & 18,00 & 1,71 & 1,98 \\
\hline & Cedrela fissilis Vell. & CL & 3 & 0,17 & 0,53 & 1,31 & 3,00 & 0,28 & 0,59 \\
\hline & Trichilia casaretti C.DC. & $\mathrm{CS}$ & 3 & 0,17 & 0,01 & 0,02 & 3,00 & 0,28 & 0,16 \\
\hline \multirow[t]{5}{*}{ Monimiaceae } & Mollinedia calodonta Perkins & $\mathrm{CS}$ & 2 & 0,12 & 0,01 & 0,03 & 2,00 & 0,19 & 0,11 \\
\hline & Mollinedia clavigera Tul. & CS & 12 & 0,69 & 0,06 & 0,15 & 10,00 & 0,95 & 0,60 \\
\hline & Mollinedia schottiana (Spreng.) Perkins & $\mathrm{CS}$ & 5 & 0,29 & 0,03 & 0,07 & 5,00 & 0,47 & 0,28 \\
\hline & Mollinedia triflora (Spreng.) Tul. & CS & 5 & 0,29 & 0,02 & 0,04 & 5,00 & 0,47 & 0,27 \\
\hline & Mollinedia uleana Perkins & CS & 1 & 0,06 & 0,01 & 0,03 & 1,00 & 0,09 & 0,06 \\
\hline \multirow[t]{4}{*}{ Moraceae } & Brosimum glaziovii Taub. & $\mathrm{CS}$ & 1 & 0,06 & 0,07 & 0,18 & 1,00 & 0,09 & 0,11 \\
\hline & Brosimum lactescens (S.Moore) C.C.Berg & CS & 9 & 0,52 & 0,10 & 0,24 & 7,00 & 0,66 & 0,48 \\
\hline & Ficus gomelleira Kunth & CS & 2 & 0,12 & 0,24 & 0,60 & 2,00 & 0,19 & 0,30 \\
\hline & Sorocea bonplandii (Baill.) W.C.Burger et al. & $\mathrm{CL}$ & 4 & 0,23 & 0,02 & 0,04 & 4,00 & 0,38 & 0,22 \\
\hline Myristicaceae & Virola bicuhyba (Schott ex Spreng.) Warb. & $\mathrm{CL}$ & 12 & 0,69 & 0,78 & 1,92 & 11,00 & 1,04 & 1,22 \\
\hline \multirow[t]{34}{*}{ Myrtaceae } & Calyptranthes grandifolia O.Berg & $\mathrm{CS}$ & 3 & 0,17 & 0,01 & 0,03 & 3,00 & 0,28 & 0,16 \\
\hline & Calyptranthes lucida Mart. ex DC. & CS & 20 & 1,16 & 0,22 & 0,55 & 15,00 & 1,42 & 1,04 \\
\hline & Calyptranthes strigipes O.Berg & CS & 9 & 0,52 & 0,19 & 0,48 & 9,00 & 0,85 & 0,62 \\
\hline & Eugenia beaurepaireana (Kiaersk.) D.Legrand & $\mathrm{CS}$ & 3 & 0,17 & 0,24 & 0,58 & 3,00 & 0,28 & 0,35 \\
\hline & Eugenia brasiliensis Lam. & CS & 2 & 0,12 & 0,01 & 0,03 & 2,00 & 0,19 & 0,11 \\
\hline & Eugenia brevistyla D.Legrand & CS & 2 & 0,12 & 0,01 & 0,02 & 2,00 & 0,19 & 0,11 \\
\hline & Eugenia cerasiflora Miq. & CS & 12 & 0,69 & 0,12 & 0,30 & 12,00 & 1,14 & 0,71 \\
\hline & Eugenia handroana D.Legrand & $\mathrm{CS}$ & 2 & 0,12 & 0,04 & 0,11 & 2,00 & 0,19 & 0,14 \\
\hline & Eugenia kleinii D.Legrand & $\mathrm{CS}$ & 3 & 0,17 & 0,01 & 0,03 & 2,00 & 0,19 & 0,13 \\
\hline & Eugenia multicostata D.Legrand & CS & 2 & 0,12 & 0,57 & 1,42 & 2,00 & 0,19 & 0,57 \\
\hline & Eugenia stigmatosa DC. & CS & 2 & 0,12 & 0,01 & 0,03 & 2,00 & 0,19 & 0,11 \\
\hline & Eugenia verticillata (Vell.) Angely & CS & 3 & 0,17 & 0,15 & 0,36 & 3,00 & 0,28 & 0,27 \\
\hline & Marlierea excoriata Mart. & CS & 3 & 0,17 & 0,05 & 0,12 & 3,00 & 0,28 & 0,19 \\
\hline & Marlierea silvatica (O.Berg) Kiaersk. & CS & 3 & 0,17 & 0,19 & 0,48 & 3,00 & 0,28 & 0,31 \\
\hline & Marlierea tomentosa Cambess. & $\mathrm{CS}$ & 4 & 0,23 & 0,01 & 0,03 & 4,00 & 0,38 & 0,21 \\
\hline & Myrcia aethusa (O.Berg) N.Silveira & $\mathrm{CS}$ & 8 & 0,46 & 0,06 & 0,15 & 7,00 & 0,66 & 0,42 \\
\hline & Myrcia brasiliensis Kiaersk. & $\mathrm{CS}$ & 9 & 0,52 & 0,23 & 0,57 & 7,00 & 0,66 & 0,58 \\
\hline & Myrcia dichrophylla D.Legrand & $\mathrm{CS}$ & 2 & 0,12 & 0,14 & 0,35 & 2,00 & 0,19 & 0,22 \\
\hline & Myrcia glabra (O.Berg) D.Legrand & $\mathrm{CS}$ & 2 & 0,12 & 0,07 & 0,17 & 2,00 & 0,19 & 0,16 \\
\hline & Myrcia guianensis (Aubl.) DC. & $\mathrm{CS}$ & 8 & 0,46 & 0,10 & 0,25 & 6,00 & 0,57 & 0,43 \\
\hline & Myrcia hebepetala DC. & CS & 1 & 0,06 & 0,003 & 0,01 & 1,00 & 0,09 & 0,05 \\
\hline & Myrcia pubipetala Miq. & CL & 4 & 0,23 & 0,22 & 0,53 & 4,00 & 0,38 & 0,38 \\
\hline & Myrcia pulchra (O.Berg) Kiaersk. & $\mathrm{CS}$ & 8 & 0,46 & 0,29 & 0,71 & 7,00 & 0,66 & 0,61 \\
\hline & Myrcia spectabilis DC. & $\mathrm{CS}$ & 2 & 0,12 & 0,01 & 0,02 & 1,00 & 0,09 & 0,08 \\
\hline & Myrcia tijucensis Kiaersk. & $\mathrm{CS}$ & 8 & 0,46 & 0,19 & 0,47 & 6,00 & 0,57 & 0,50 \\
\hline & Myrciaria floribunda (H.West ex Willd.) O.Berg & CS & 3 & 0,17 & 0,05 & 0,12 & 3,00 & 0,28 & 0,19 \\
\hline & Myrciaria plinioides D.Legrand & $\mathrm{CS}$ & 3 & 0,17 & 0,01 & 0,03 & 1,00 & 0,09 & 0,10 \\
\hline & Myrciaria tenella (DC.) O.Berg & $\mathrm{CS}$ & 1 & 0,06 & 0,003 & 0,01 & 1,00 & 0,09 & 0,05 \\
\hline & Não Identificada 1 & $\mathrm{CS}$ & 1 & 0,06 & 0,003 & 0,01 & 1,00 & 0,09 & 0,05 \\
\hline & Não Identificada 2 & CS & 1 & 0,06 & 0,004 & 0,01 & 1,00 & 0,09 & 0,05 \\
\hline & Não Identificada 3 & $\mathrm{CS}$ & 1 & 0,06 & 0,01 & 0,01 & 1,00 & 0,09 & 0,06 \\
\hline & Neomitranthes glomerata (D.Legrand) D.Legrand & $\mathrm{CS}$ & 5 & 0,29 & 0,03 & 0,08 & 5,00 & 0,47 & 0,28 \\
\hline & Pimenta pseudocaryophyllus (Gomes) Landrum & $\mathrm{CS}$ & 1 & 0,06 & 0,01 & 0,04 & 1,00 & 0,09 & 0,06 \\
\hline & Plinia pseudodichasiantha (Kiaersk.) G.M.Barroso ex Sobral & $\mathrm{CS}$ & 4 & 0,23 & 0,02 & 0,06 & 4,00 & 0,38 & 0,22 \\
\hline \multirow[t]{2}{*}{ Nyctaginaceae } & Guapira opposita (Vell.) Reitz & $\mathrm{CS}$ & 8 & 0,46 & 0,25 & 0,62 & 7,00 & 0,66 & 0,58 \\
\hline & Pisonia ambigua Heimerl & CS & 1 & 0,06 & 0,02 & 0,04 & 1,00 & 0,09 & 0,06 \\
\hline Ochnaceae & Ouratea parviflora (A.DC.) Baill. & CS & 2 & 0,12 & 0,01 & 0,01 & 2,00 & 0,19 & 0,11 \\
\hline Olacaceae & Heisteria silvianii Schwacke & $\mathrm{CS}$ & 12 & 0,69 & 0,35 & 0,86 & 12,00 & 1,14 & 0,90 \\
\hline Peraceae & Pera glabrata (Schott) Poepp. ex Baill. & CL & 3 & 0,17 & 0,04 & 0,09 & 3,00 & 0,28 & 0,18 \\
\hline \multirow[t]{2}{*}{ Phyllanthaceae } & Hieronyma alchorneoides Allemão & $\mathrm{CL}$ & 12 & 0,69 & 0,73 & 1,80 & 12,00 & 1,14 & 1,21 \\
\hline & Richeria grandis Vahl & $\mathrm{CS}$ & 1 & 0,06 & 0,07 & 0,18 & 1,00 & 0,09 & 0,11 \\
\hline
\end{tabular}

FLORESTA, Curitiba, PR, v. 45, n. 3, p. 555 - 566, jul. / set. 2015.

Maçaneiro, J. P. de; Seubert, R. C.; Schorn, L. A.

ISSN eletrônico 1982-4688 / ISSN impresso 0015-3826 


\begin{tabular}{|c|c|c|c|c|c|c|c|c|c|}
\hline Podocarpaceae & Podocarpus sellowii Klotzsch ex Endl. & $\mathrm{CS}$ & 6 & 0,35 & 0,10 & 0,24 & 5,00 & 0,47 & 0,35 \\
\hline Polygonaceae & Coccoloba warmingii Meisn. & $\mathrm{CL}$ & 2 & 0,12 & 0,01 & 0,02 & 2,00 & 0,19 & 0,11 \\
\hline \multirow{2}{*}{ Primulaceae } & Cybianthus brasiliensis (Mez) G.Agostini & $\mathrm{CL}$ & 3 & 0,17 & 0,01 & 0,02 & 3,00 & 0,28 & 0,16 \\
\hline & $\begin{array}{l}\text { Myrsine hermogenesii (Jung-Mend. \& } \\
\text { Bernacci) M.F.Freitas \& Kin.-Gouv. }\end{array}$ & $\mathrm{CS}$ & 3 & 0,17 & 0,02 & 0,05 & 2,00 & 0,19 & 0,14 \\
\hline Quiinaceae & Quiina glaziovii Engl. & $\mathrm{CS}$ & 4 & 0,23 & 0,09 & 0,22 & 3,00 & 0,28 & 0,25 \\
\hline \multirow[t]{8}{*}{ Rubiaceae } & Amaioua guianensis Aubl. & $\mathrm{CS}$ & 37 & 2,14 & 0,57 & 1,41 & 28,00 & 2,66 & 2,07 \\
\hline & Bathysa australis (A.St.-Hil.) K.Schum. & $\mathrm{CS}$ & 5 & 0,29 & 0,17 & 0,42 & 4,00 & 0,38 & 0,36 \\
\hline & Cordiera concolor (Cham.) Kuntze & $\mathrm{CL}$ & 4 & 0,23 & 0,02 & 0,05 & 4,00 & 0,38 & 0,22 \\
\hline & $\begin{array}{l}\text { Faramea montevidensis (Cham. \& Schltdl.) } \\
\text { DC. }\end{array}$ & $\mathrm{CS}$ & 12 & 0,69 & 0,14 & 0,34 & 12,00 & 1,14 & 0,72 \\
\hline & Psychotria carthagenensis Jacq. & $\mathrm{CL}$ & 5 & 0,29 & 0,12 & 0,31 & 5,00 & 0,47 & 0,36 \\
\hline & Psychotria nemorosa Gardner & $\mathrm{CS}$ & 1 & 0,06 & 0,002 & 0,01 & 1,00 & 0,09 & 0,05 \\
\hline & Rudgea jasminoides (Cham.) Müll.Arg. & $\mathrm{CS}$ & 3 & 0,17 & 0,01 & 0,02 & 3,00 & 0,28 & 0,16 \\
\hline & Rudgea recurva Müll.Arg. & $\mathrm{CS}$ & 34 & 1,97 & 0,13 & 0,32 & 24,00 & 2,28 & 1,52 \\
\hline Rutaceae & Esenbeckia grandiflora Mart. & CS & 5 & 0,29 & 0,08 & 0,20 & 5,00 & 0,47 & 0,32 \\
\hline Sabiaceae & Meliosma sellowii Urb. & $\mathrm{CS}$ & 1 & 0,06 & 0,004 & 0,01 & 1,00 & 0,09 & 0,05 \\
\hline Salicaceae & Casearia obliqua Spreng. & CL & 1 & 0,06 & 0,04 & 0,09 & 1,00 & 0,09 & 0,08 \\
\hline Sapindaceae & Matayba intermedia Radlk. & $\mathrm{CL}$ & 7 & 0,41 & 0,30 & 0,74 & 6,00 & 0,57 & 0,57 \\
\hline \multirow[t]{2}{*}{ Sapotaceae } & Chrysophyllum viride Mart. \& Eichler & $\mathrm{CS}$ & 1 & 0,06 & 0,03 & 0,06 & 1,00 & 0,09 & 0,07 \\
\hline & Pouteria venosa (Mart.) Baehni & $\mathrm{CS}$ & 4 & 0,23 & 0,26 & 0,65 & 4,00 & 0,38 & 0,42 \\
\hline Theaceae & Laplacea fructicosa (Schrad.) Kobuski & CL & 1 & 0,06 & 0,02 & 0,04 & 1,00 & 0,09 & 0,06 \\
\hline \multirow{2}{*}{ Urticaceae } & Cecropia glaziovii Snethl. & $\mathrm{P}$ & 5 & 0,29 & 0,04 & 0,10 & 4,00 & 0,38 & 0,26 \\
\hline & Pourouma guianensis Aubl. & $\mathrm{CL}$ & 5 & 0,29 & 0,09 & 0,22 & 5,00 & 0,47 & 0,33 \\
\hline Total & & & 1.727 & 100,00 & 40,39 & 100,0 & $1.054,00$ & 100,00 & 100,00 \\
\hline
\end{tabular}

GE: grupo ecológico; P: pioneira; CL: clímax exigente de luz; CS: clímax tolerante à sombra; DA: densidade absoluta (ind.ha $\left.{ }^{-1}\right)$; DR: densidade relativa (\%); FA: frequência absoluta (\%); FR: frequência relativa (\%); DoA: dominância absoluta $\left(\mathrm{m}^{2} . \mathrm{ha}^{-1}\right)$; DoR: dominância relativa (\%); PI: percentual de importância (\%).

$\mathrm{Na}$ análise de agrupamentos, encontrou-se um valor de 0,91 para o coeficiente de correlação cofenética do dendrograma, indicando que $91 \%$ da matriz de dados originais foram representados na forma de dendrograma (Figura 1). Além disso, verificou-se correlação altamente significativa entre a matriz de distância e a matriz cofenética (Mantel, $P<0,001$ ). A composição de espécies entre os trabalhos analisados diferiu conforme a distância geográfica dos locais (Tabela 2), indicando a formação de dois grupos florísticos. O grupo I foi formado pelos trabalhos realizados na região sul-catarinense e o grupo II pelos trabalhos realizados na região do Vale do Itajaí. Observou-se que o trabalho de Negrelle (2006), que é constituído por uma Floresta Pluvial Subtropical de Baixada, não se agrupou com os demais trabalhos, revelando se tratar de um local com composição de espécies distinta.

Tabela 2. Relação dos 12 trabalhos utilizados nas análises de similaridade florística da Floresta Pluvial Subtropical no Sul do Brasil.

Table 2. Listing of the 12 studies considered in the floristic similarity analysis of a Subtropical Rain Forest in southern Brazil.

\begin{tabular}{lccccccc}
\hline Município & Autor & Longitude & Latitude & Elevação (m) & Método & Área (ha) & $\begin{array}{c}\text { DAP } \\
\text { (cm) }\end{array}$ \\
\hline Blumenau & Vibrans (1999) & 684734 & 7024053 & $28-34$ & Parcelas & 1,00 & 5,0 \\
& Sevegnani (2003) & 691222 & 7020859 & $35-135$ & Parcelas & 1,00 & 5,0 \\
& Ghoddosi (2005) & 691066 & 7020847 & $35-135$ & Parcelas & 1,00 & 4,8 \\
Brusque & Presente estudo & 709195 & 7000878 & $52-161$ & Parcelas & 1,00 & 5,0 \\
Criciúma & Silva (2006) & 653155 & 6825320 & 34 & Parcelas & 1,00 & 5,0 \\
Ilhota & Iza (2002) & 704842 & 7033731 & $372-489$ & Parcelas & 1,00 & 5,0 \\
Itapoá & Negrelle (2006) & 735626 & 7112928 & 9 & Parcelas & 1,00 & 5,0 \\
Orleans & Citadini-Zanette (1995) & 668251 & 6862826 & 145 & Parcelas & 1,00 & 5,0 \\
Siderópolis & Colonetti et al. (2009) & 639390 & 6835699 & 178 & Parcelas & 1,00 & 5,0 \\
& Martins (2005) & 656022 & 6836937 & 170 & Parcelas & 1,00 & 5,0 \\
Timbé do Sul & Martins (2010) & 613188 & 6821129 & 441 & Parcelas & 1,00 & 5,0 \\
Turvo & Emerich (2009) & 628129 & 6800492 & 32 & Parcelas & 1,00 & 5,0 \\
\hline
\end{tabular}

No Escalonamento Multidimensional Não Métrico (NMDS), a composição de espécies nos trabalhos também diferiu conforme a distância entre os locais, indicando a formação de dois grupos 
(Figura 2). Os dois primeiros eixos de ordenação segregaram os trabalhos da região sul-catarinense e do Vale do Itajaí e produziram $76,7 \%$ da correlação entre as distâncias de ordenação e o espaço ndimensional original. O stress médio obtido para os dados reais $(11,37)$ e aleatorizados $(20,18)$ dos dois primeiros eixos de ordenação se mantiveram estáveis na porção final das iterações e apresentaram significância estatística pelo teste de Monte Carlo $(P \leq 0,05)$. Além disso, os dois grupos florísticos formados pelo NMDS e pela análise de agrupamentos apresentaram diferenças altamente significativas entre si (ANOSIM, $P<0,001$ ).

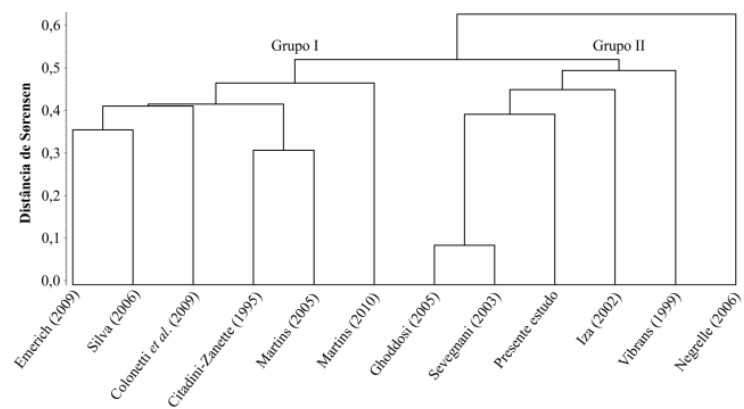

Figura 1. Dendrograma de classificação construído através de uma matriz de presença e ausência de espécies para 12 trabalhos realizados na Floresta Pluvial Subtropical de Santa Catarina.

Figure 1. Classification dendrogram constructed by a presence and absence species matrix gathered from 12 studies conducted in Subtropical Rain Forest of Santa Catarina.

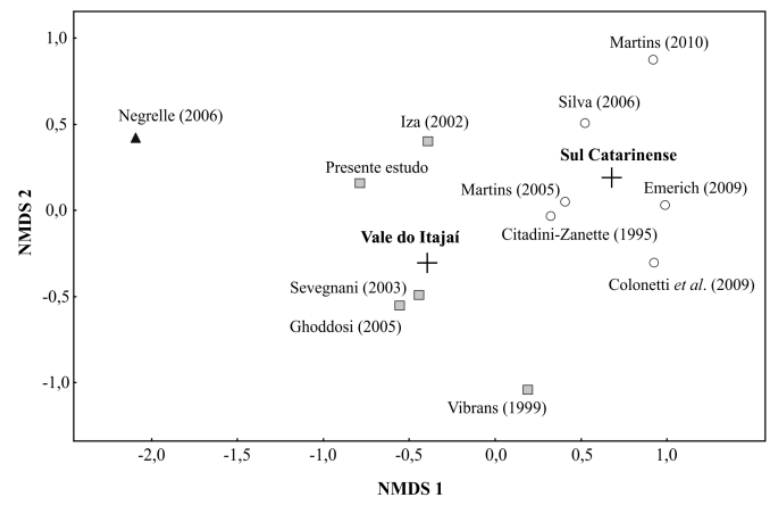

Figura 2. Diagrama de ordenação produzido pelo Escalonamento Multidimensional Não Métrico (NMDS) através de uma matriz de presença e ausência de espécies para 12 trabalhos realizados na Floresta Pluvial Subtropical de Santa Catarina.

Figure 2. Ordination diagram produced by the Non Multidimensional Scaling (NMDS) using a presence and absence species matrix gathered from 12 studies conducted in Subtropical Rain Forest of Santa Catarina.

A diferença marcante entre a composição de espécies dos grupos observados pode indicar a influência da distância geográfica entre os locais. Ruokolainen e Tuomisto (2002) verificaram que a distância geográfica pode refletir na limitação de dispersão e proporcionar mudanças na composição de espécies em florestas tropicais. Além disso, a latitude é uma variável ambiental que condiciona, de forma indireta, mudanças nos padrões de composição de espécies da Mata Atlântica (OLIVEIRA-FILHO; FONTES, 2000). Oliveira-Filho et al. (2013), por exemplo, verificaram que a latitude exerce papel importante nas mudanças da composição de espécies da Mata Atlântica no Sul do Brasil. Esses autores verificaram que a riqueza de espécies diminui conforme o aumento da latitude. No presente estudo, as diferenças entre a composição de espécies nos grupos florísticos podem estar relacionadas com a distância geográfica dos locais. 
Por outro lado, fatores ambientais relacionados a solo e relevo, estado de conservação das florestas e tipo de formação florestal existente também podem ser considerados como importantes para explicar a segregação dos grupos florísticos (SCHEER; BLUM, 2011). Na Floresta Pluvial Subtropical de Santa Catarina, por exemplo, alguns estudos já apontaram que a composição de espécies entre diferentes formações florestais apresenta diferenciações marcantes (KLEIN, 1980; MARTINS, 2010; LINGNER et al., 2013). Esses estudos apontaram que o principal fator ambiental que condiciona alterações na composição de espécies está associado à cota altimétrica. Assim, no presente estudo, o tipo de formação florestal existente entre os locais comparados pode estar influenciando na segregação dos grupos florísticos.

\section{CONCLUSÕES}

- A floresta estudada encontra-se em bom estado de conservação, uma vez que foram registradas apenas quatro espécies pioneiras e as famílias com maior riqueza de espécies foram Myrtaceae, Lauraceae e Fabaceae, com destaque para os gêneros Ocotea, Myrcia e Eugenia.

- Entre as principais espécies que caracterizaram a estrutura da floresta, estão E. edulis, S. guianensis, $O$. aciphylla, $T$. guianensis e $V$. compacta, sendo que a primeira delas apresentou aspecto de dominante na área de estudo, principalmente pelos seus elevados valores de densidade e frequência.

- Os resultados indicaram a formação de dois grupos florísticos entre os trabalhos comparados, sendo o primeiro deles composto por trabalhos realizados na região sul-catarinense e o segundo por trabalhos realizados na região do Vale do Itajaí.

\section{AGRADECIMENTOS}

À Coordenação de Aperfeiçoamento de Pessoal de Nível Superior (CAPES), pela concessão da bolsa de estudos ao primeiro autor, e ao Programa de Pós-Graduação em Engenharia Florestal da Universidade Regional de Blumenau (FURB), pelo auxílio financeiro prestado para execução desta pesquisa.

\section{REFERÊNCIAS}

APG III - The Angiosperm Phylogeny Group III. An update of the Angiosperm Phylogeny Group classification for the ordens and families of flowering plants: APG III. Botanical Journal of the Linnean Society, v. 161, p. 105 - 121, 2009.

CITADINI-ZANETTE, V. Florística, fitossociologia e aspectos da dinâmica de um remanescente de Mata Atlântica na microbacia do rio Novo, Orleans, SC. 249 f. Tese (Doutorado em Ecologia) Universidade Federal de São Carlos, São Carlos, 1995.

CLARKE, K. R. Non-parametric multivariate analysis of changes in community structure. Australian Journal of Ecology, v. 18, p. 117 - 143, 1993.

COLONETTI, S.; CITADINI-ZANETTE, V.; MARTINS, R.; SANTOS, R.; ROCHA, E.; JARENKOW, J. A. Florística e estrutura fitossociológica em floresta ombrófila densa submontana na barragem do rio São Bento, Siderópolis, estado de Santa Catarina. Acta Scientiarum Biological Sciences, v. 31, p. 397 405, 2009.

EMPRESA BRASILEIRA DE PESQUISA AGROPECUÁRIA (EMBRAPA). Solos do Estado de Santa Catarina. Rio de Janeiro. 2004. 721 p.

EMERICH, K. H. Composição florística e relação entre variáveis ambientais e a estrutura da comunidade arbórea de fragmento florestal ciliar do rio Turvo, município de Turvo, Santa Catarina. 81 f. Dissertação (Mestrado em Ciências Ambientais) - Universidade do Extremo Sul Catarinense, Criciúma, 2009.

EMPRESA DE PESQUISA AGROPECUÁRIA E EXTENSÃO RURAL DE SANTA CATARINA (EPAGRI). Atlas Climatológico do Estado de Santa Catarina. Florianópolis. 1 CD. 2002. 
FORZZA, R. C.; BAUMGRATZ, J. F. A.; BICUDO, C. E. M.; CANHOS, D. A. L.; CARVALHO JR., A. A.; COELHO, M. A. N.; COSTA, A. F.; COSTA, D. P.; HOPKINS, M. G.; LEITMAN, P. M.; LOHMANN, L. G.; LUGHADHA, E. N.; MAIA, L. C.; MARTINELLI, G.; MENEZES, M.; MORIM, M. P.; PEIXOTO, A. L.; PIRANI, J. R.; PRADO, J.; QUEIROZ, L. P.; SOUZA, S.; SOUZA, V. C.; STEHMANN, J. R.; SYLVESTRE, L. S.; WALTER, B. M. T.; ZAPPI, D. C. New brazilian floristic list highlights conservation challenges. BioScience, v. 62, p. 39 - 45, 2012.

FORZZA, R. C.; STEHMANN, J. R.; NADRUZ, M. Lista de Espécies da Flora do Brasil. Jardim Botânico do Rio de Janeiro. <Disponível em: http://floradobrasil.jbrj.gov.br/>. Acesso em: 12/10/2014.

GHODDOSI, S. M. Dinâmica do componente arbóreo (1999-2004) de um trecho de Floresta Ombrófila Densa em Blumenau, SC. 140 f. Dissertação (Mestrado em Engenharia Ambiental) Universidade Regional de Blumenau, Blumenau, 2005.

GIULIETTI, A. M.; HARLEY, R. M.; QUEIROZ, L. P.; WANDERLEY, M. G. L.; VAN DEN BERG, C. Biodiversity and Conservation of Plants in Brazil. Conservation Biology, v. 19, p. 632 - 639, 2005.

IZA, O. B. Parâmetros de autoecologia de uma comunidade arbórea de Floresta Ombrófila Densa, no Parque Botânico do Morro do Baú, Ilhota, SC. 92 f. Dissertação (Mestrado em Biologia Vegetal) Universidade Federal de Santa Catarina, Florianópolis, 2002.

KLEIN, R. M. Ecologia da flora e vegetação do Vale do Itajaí. Sellowia, v. 32, p. 165 - 389, 1980.

LEGENDRE, P.; LEGENDRE, L. Numerical Ecology. Amsterdan: Elsevier, 2012. 853 p.

LINGNER, D. V.; SCHORN, L. A.; VIBRANS, A. C.; MEYER, L.; SEVEGNANI, L.; GASPER, A. L.; SOBRAL, M. G.; KRÜGER, A.; KLEMZ, G.; SCHMIDT, R.; ANASTÁCIO-JÚNIOR, C. Fitossociologia do componente arbóreo/arbustivo da Floresta Ombrófila Densa em Santa Catarina. In: VIBRANS, A. C.; SEVEGNANI, L.; GASPER, A. L.; LINGNER, D. V. Inventário Florístico Florestal de Santa Catarina: Floresta Ombrófila Densa. Blumenau, 2013, p. 159 - 200.

MANTEL, N. The detection of disease clustering and a generalized regression approach. Cancer Research, v. 27, p. 209 - 220, 1967.

MARTINS, R. Florística, estrutura fitossociológica e interações interespecíficas de um remanescente de Floresta Ombrófila Densa como subsídio para recuperação de áreas degradadas pela mineração de carvão, Siderópolis, SC. 93 f. Dissertação (Mestrado em Biologia Vegetal) - Universidade Federal de Santa Catarina, Florianópolis, 2005.

Composição e estrutura vegetacional em diferentes formações na Floresta Atlântica, sul de Santa Catarina, Brasil. 148 f. Tese (Doutorado em Botânica) - Universidade Federal do Rio Grande do Sul, Porto Alegre, 2010.

McCUNE, B.; GRACE, J. B. Analysis of ecological communities. MjM: Gleneden Beach, 2002. 304 p. MINISTÉRIO DO MEIO AMBIENTE (MMA). Portaria no 443, de 17 de dezembro de 2014. Disponível em: <http://www.ibama.gov.br/documentos/lista-de-especies-ameacadas-de-extincao>. Acesso em: 13/02/2015.

MORO, M. F.; MARTINS, F. R. Métodos de levantamento do componente arbóreo-arbustivo. In: FELFILI, J. M.; EISENLOHR, P. V.; MELO, M. M. R. F.; ANDRADE, L. A.; NETO, J. A. A. M. Fitossociologia no Brasil: métodos e estudos de casos. Viçosa: Editora UFV, 2011. p. 174 - 212.

MUELLER-DOMBOIS, D.; ELLENBERG, H. Aims and methods of vegetation ecology. New Jersey: The Blackburn Press, 2002. 547 p.

MYERS, N.; MITTERMEIER, R. A.; MITTERMEIER, C. G.; FONSECA, G. A. B.; JENT, J. Biodiversity hotspots for conservation priorities. Nature, v. 403, p. 853 - 858, 2000.

NEGRELLE, R. R. B. Composição florística e estrutura vertical de um trecho de Floresta Ombrófila Densa de planície quaternária. Hoehnea, v. 33, p. 261 - 289, 2006. 
OLIVEIRA-FILHO, A. T. Estudos ecológicos da vegetação como subsídios para programas de revegetação com espécies nativas: uma proposta metodológica. Cerne, v. 1, p. 64 - 72, 1994.

OLIVEIRA-FILHO, A. T.; FONTES, M. A. Patterns of floristic differentiation among Atlantic Forests in Southeastern Brazil and the influence of climate. Biotropica, v. 32, p. 793 - 810, 2000.

OLIVEIRA-FILHO, A. T. Classificação das fitofisionomias da América do Sul cisandina tropical e subtropical: proposta de um novo sistema - prático e flexível - ou uma injeção a mais de caos? Rodriguésia, v. 60, p. 237 - 258, 2009.

OLIVEIRA-FILHO, A. T.; BUDKE, J. C.; JARENKOW, J. A.; EISENLOHR, P. V.; NEVES, D. R. M. Delving into the variations in tree species composition and richness across South American subtropical Atlantic and Pampean forests. Journal of Plant Ecology, v. 6, p. 1 - 23, 2013.

PHILIPP, R. P.; MALLMANN, G.; BITENCOURT, M. F.; SOUZA, E. R.; SOUZA, M. M. A.; LIZ, J. D.; WILD, F.; ARENDT, S.; OLIVEIRA, A. S.; DUARTE, L.; RIVERA, C. B.; PRADO, M. Caracterização litológica e evolução metamórfica da porção leste do Complexo Metamórfico Brusque, Santa Catarina. Revista Brasileira de Geociências, v. 34, p. 21 - 34, 2004.

RUOKOLAINEN, K.; TUOMISTO, H. Beta-diversity in tropical trees. Science, v. 297, p. 1439, 2002.

SCHEER, M. B.; BLUM, C. T. Arboreal diversity of the Atlantic Forest of Southern Brazil: From the beach ridges to the Paraná River. In: GRILLO, O.; VENORA, G. The Dynamical Processes of Biodiversity Case Studies of Evolution and Spatial Distribution. Rijeka: Intech. 2011. p. 110 - 134.

SCHORN, L. A.; GALVÃO, F. Dinâmica do estrato arbóreo em três estádios sucessionais de uma Floresta Ombrófila Densa em Blumenau, SC. Cerne, v. 15, p. 221 - 235, 2009.

SCHUlZ-JÚNIOR, A.; ALBUQUERQUE, L. F. F. Geologia da quadrícula Rio do Sul, Santa Catarina. DNPM, Porto Alegre. 1969. 109 p.

SEVEGNANI, L. Dinâmica de população de Virola bicuhyba (Schott) Warb. (Myristicaceae) e estrutura fitossociológica de Floresta Pluvial Atlântica, sob clima temperado úmido de verão quente, Blumenau, SC. 161 f. Tese (Doutorado em Ecologia) - Universidade de São Paulo, São Paulo, 2003.

SILVA, R. T. Florística e estrutura da sinúsia arbórea de um fragmento urbano de Floresta Ombrófila Densa do município de Criciúma, Santa Catarina. 61 f. Dissertação (Mestrado em Ciências Ambientais) - Universidade do Extremo Sul Catarinense, Criciúma, 2006.

TABARELli, M.; MANTOVANI, W. Clareiras naturais e a riqueza de espécies pioneiras em uma Floresta Atlântica Montana. Revista Brasileira de Biologia, v. 59, p. 251 - 261, 1999.

THE PLANT LIST. The Angiosperms (Flowering plants). Disponível em: <http://www.theplantlist.org>. Acesso em: 08/09/2014.

THOMAS, W. W.; CARVAlHO, A. M. V.; AMORIM, A. M. A.; GARRISON, J.; ARBELÁEZ, A. L. Plant endemism in two forests in southern Bahia, Brasil. Biodiversity and Conservation, v. 7, p. 311 322, 1998.

VELOSO, H. P.; KLEIN, R. M. Dinamismo e fidelidade das espécies em associações do município de Brusque, Estado de Santa Catarina. Sellowia, v. 10, p. 9 - 124, 1959.

VIBRANS, A. C. Subsídios para o manejo de uma floresta secundária no Salto Weissbach, Blumenau, SC. 103 f. Dissertação (Mestrado em Engenharia Ambiental) - Universidade Regional de Blumenau, Blumenau, 1999.

VIBRANS, A. C.; McROBERTS, R. E.; MOSER, P.; NICOLETTI, A. L. Using satellite image-based maps and ground inventory data to estimate the area of the remaining Atlantic forest in the Brazilian state of Santa Catarina. Remote Sensing of Environment, v. 130, n. 1, p. 87 - 95, 2013. 